\title{
Modelling the Reduced Coefficient of Volumetric Rigidity in High-Pressure Rubber-Cord Shell Hoses
}

\author{
Aleksandr Rybak $^{1}$ and Olga Lyakhnitskaya ${ }^{1, *}$ \\ ${ }^{1}$ Don State Technical University, Rostov-on-Don, Russian Federation
}

\begin{abstract}
In recent years, methods for calculating the hydraulic power drive have become increasingly widespread, taking into account the violation of the continuity equation during its operation at unsteady modes due to the use of the theory of volumetric rigidity in modeling. In this article approach to calculation of the reduced coefficient of volume rigidity of high-pressure hoses is considered.
\end{abstract}

\section{Introduction}

Hydraulic systems are increasingly used in modern machines and mechanisms. Typically, the working fluid in such systems operates under high pressure. To transport this liquid, metal pipelines and high-pressure hoses (HPH) are used. The working fluid can be various kinds of mineral and synthetic oils, liquid fuels, water-oil emulsions or water.

One of the most important characteristics of a hydraulic drive is the quality of its operation at nonsteady-state conditions, when the equation of continuity of flow is violated because of the compressibility of the working fluid and the deformation of the hydraulic system [1-3]. In this case, in modelling, a certain dummy flow of fluid is introduced into the equation of continuity, which compensates for these effects.

However, in recent years, another method of modelling hydraulic power drives has been increasingly used, to take account of the violation of the continuity equation during its operation at unsteady modes in modelling - the theory of volumetric rigidity [4,5].

Thus, instead of the reduced modulus of elasticity of the system, the reduced coefficient of its volume rigidity is used $[6,7]$. Several formalized dependencies have been obtained to determine the reduced coefficient of volume hardness of various hydraulic devices [8-15]. However, for rubber-cord shell high-pressure hoses, such dependencies have not yet been obtained, since their elastic properties still do not lend themselves to a rigorous analytical description. Here, we address this omission.

\section{Theory}

The volumetric rigidity of a hydraulic drive is the property of the hydraulic system to resist changing its internal volume when the pressure changes. It is characterized by "reduced coefficient of volumetric rigidity" of a system.
The given volume rigidity will allow us to judge pliability of system in general and each her element separately similar to a concept of rigidity of solid bodies which indicates a change of the linear sizes of elastic elements at impact on them of external forces [1].

Liquid compresses when forcing in system with increased pressure that imposes the influence on property of the system in general. Therefore, the "volume rigidity" characterized by a coefficient of volumetric rigidity may serve as an integral criterion of dynamic properties of hydraulic system considering that change of volume of hydraulic system results from the increase liquid pressure boost that at the same time compresses [6].

$$
\mathrm{C}_{\text {пр }}=-\frac{d p}{d V},
$$

where $\mathrm{C}_{\text {пр }}$ - the reduced coefficient of volumetric rigidity of the considered site of the hydraulic actuator; $d p$ - pressure increment of the considered site; $d V$-interior content increment of the considered site corresponding to an increment of pressure upon $d p$.

We will define coefficient of volumetric rigidity of plant and equipment, hydraulic actuator as pressure increment caused by additional injection of a unit of volume, power liquid given to power liquid condition in hydraulic reservoir and environmental condition.

In addition to metal pipelines, high-pressure hoses (HPH) are also in current use. They are characterised by being composed of a rubber-cord shell and are used in the machine-building industry, in agriculture, in various types of mining and mining machines, in road-building machinery and in the metallurgical industry and other industries. They are also used in vehicles as power steering hoses and brake hoses. HPHs are thus essential to a wide range of different applications The technique and experimental investigations made for a narrow class of high pressure hoses [16-18], but a general mathematical formula, suitable for calculating the reduced volumetric coefficient of stiffness of any type and size do not exist. The aim of this work is to find the single formula for

\footnotetext{
Corresponding author: lolyv@yandex.ru
} 
calculating the reduced volumetric stiffness of any high pressure hoses.

\section{Analysis}

According to GOST 6286-73 [19] the high-pressure hoses depending on the strength of the wire used, must be made in three groups: A, B and B.

Group A is manufactured with the use of a wire with a breaking force of not less than $15 \mathrm{~kg}$.

Group B is manufactured using a wire with a breaking force of not less than $17.5 \mathrm{~kg}$.

Group B is manufactured using a wire with a breaking force of at least $20 \mathrm{~kg}$.

Group $\mathrm{Z}$ should are made of a lattin wire and a breaking force of at least $20 \mathrm{~kg}$.

The high-pressure hoses of each group, depending on the design, can be of two types:

Type I- with one metal braiding (fig. 1)

Group Z

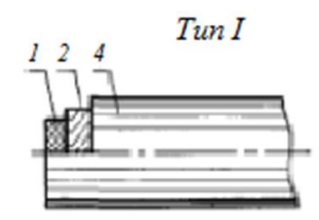

Group A, B, C

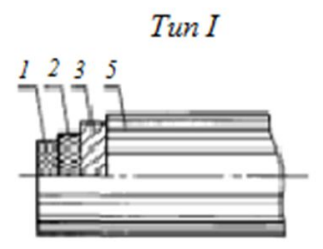

Fig. 1.The high-pressure hoses type I construction with metallic braidings. $\mathrm{Z}$ group: 1 - hose tube; 2 - metal braiding; 3 - squeegee; 4 - outer rubber layer. A, B, C group: 1 - hose tube; 2 - textile braiding; 3 - metal braiding; 4 - provisional rubber layer; 5 - outer rubber layer.

Type II- with two metal braiding (fig. 2)

Group Z

Group A, B, C
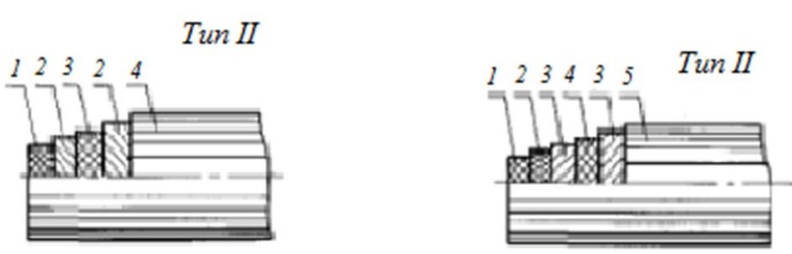

Fig. 2. The high-pressure hoses type II construction with metallic braidings. $\mathrm{Z}$ group: 1 - hose tube; 2 - metal braiding; 3 - squeegee; 4 - outer rubber layer. A, B, C group: 1 - hose tube; 2 - textile braiding; 3 - metal braiding; 4 - provisional rubber layer; 5 - outer rubber layer.

In the HPH, the first layer (the inner rubber hose chamber), which is firmly connected to the frame, receives a radially directed hydrostatic pressure $\left(p_{1}\right)$ applied to the surface of radius $\left(r_{1}\right)$. On the outer surface of the chamber of radius $\left(r_{2}\right)$, there is a pressure $\left(p_{2}\right)$ arising from the action of the frame. The rubber chamber is formed from a material with a Poisson's ratio close to 0.5 [4]. The second frame ply, along its inner surface of radius $\left(r_{2}\right)$, experiences a radially directed hydrostatic pressure $\left(p_{2}\right)$. On the outer surface of the chamber, of radius $r_{3}$, there is a pressure $p_{3}$. The last pressure is assumed equal to 0 , thus excluding the influence of the third layer-the external rubber lining. From the assumptions made, a calculation formula can be obtained for a single-loop $\mathrm{HPH}$ :

$$
p_{1}=0.184 \cdot K_{B} \cdot N \cdot n \cdot \frac{1}{\left(r+r_{1}\right)^{2}}
$$

where $K_{B}$ - braid durability to a break-up of N/thread, N - quantity of the streams of a wire put by the spool of the braiding machine, $\mathrm{n}$ - number of wires in the spool.

The second layer is the frame consisting of a row concentric or spirulate located laying which elements have some possibility of a shift, unlike the first layer. The special layer and structural design represent in homogeneous and anisotropic material.

We will accept the following assumptions for simplification of calculations:

1. Forces arising in a hose wall are perceived generally by a frame as the most rigid part of a hose;

2. The frame is considered as thin-walled cylinder closed from edges the outer rubber cover isn't taken into consideration;

3. The outer rubber cover is not taken into consideration. According to Sukharev and Lepetov [20] the significant difference in the loadings perceived by the first and last braidings is revealed. They represented the distribution of loads in the frame of a hose having $i$ homogeneous braidings in the form:

$$
p=p_{1}+p_{2}+p_{3}+\cdots+p_{i},
$$

where $p_{1}, p_{2}, p_{3}, \ldots, p_{i}$ are the fractions of the hydraulic pressure $p$ perceived by the corresponding metal braidings.

The equation of joint deformations takes the form:

$$
p \cdot r_{1}^{2}=p_{1 \_2} \cdot\left(r_{2}^{2}+2 r_{1}^{2}\right),
$$

where $r_{1}$ and $r_{2}$ are the average calculated diameters of the overlap of the first and second braidings (in centimeters), and $p_{12}$ is the intermediate pressure between the first and second braidings $\left(10^{5} \mathrm{~Pa}\right)$.

It follows from (2) that

or

$$
p_{1}=p-p_{1 \_} 2
$$

$$
p_{2}=p_{1 \_2}
$$

From here:

$$
\begin{gathered}
p_{1}=p \cdot\left(1-\frac{r_{1}^{2}}{r_{2}^{2}+2 r_{1}^{2}}\right) \\
p_{2}=p \cdot\left(\frac{r_{1}^{2}}{r_{2}^{2}+2 r_{1}^{2}}\right)
\end{gathered}
$$

Expressing $p_{2}$ in terms of $p_{1}$, we obtain the calculated formula for finding $p_{2}$ :

$$
p_{2}=p_{1} \cdot\left(\frac{r_{1}^{2}}{r_{2}^{2}+2 r_{1}^{2}}\right)
$$


Using formula (2), we thus obtain a formula for calculating the pressure $p_{2}$ :

$$
p_{2}=0.184 \cdot k_{B} \cdot N \cdot n \cdot\left(\frac{1}{\left(r+r_{1}\right)^{2}}+\frac{1}{r_{1}^{2}+r_{2}^{2}}\right) \cdot \frac{C_{1}}{C_{2}^{2}}
$$

A variety of correction coefficient is introduced for correction of deviations hose multilayered frame from conditions in thin-walled isotropic covers and for accounting of strength and deformation properties of the material of a frame. We will enter $\mathrm{C} 1$ and $\mathrm{C} 2$ coefficients for braided HPH [20]. The multiplier $\mathrm{C}_{1}$ considers nonsimultaneity of a gap in parallel going braidings in a stream:

$$
\mathrm{C}_{1}=1-0,015(n-1),
$$

and multiplier $\mathrm{C} 2$ - elongation $\varepsilon$ wire material in braid:

$$
\mathrm{C}_{2}=1+\frac{\varepsilon}{l}
$$

Hose process pressures containing $\mathrm{i}$ of braidings are calculated in the same way.

\section{Results}

Calculation data may be significantly simplified if conditionally to assume that in thin layer braidings of value of superimposing of average radii are close among themselves, in other word $r_{1}=r_{2}=\cdots=r_{i}$ equation (2) will become:

$$
p_{i}=0.184 \cdot K_{B} \cdot N \cdot n \cdot \sum_{1}^{i} \frac{1}{2^{i+1} \cdot\left(r+r_{1}\right)^{2}}
$$

We will receive the generalized formula for $\mathrm{HPH}$ containing $\mathrm{i}$ of braidings, having entered coefficient

$$
\begin{gathered}
\theta_{i}=\frac{0.735}{2^{i+1}} \\
p_{i}=\theta_{i} \cdot K_{B} \cdot N \cdot n \cdot \frac{1}{\left(r+r_{1}\right)^{2}} \cdot \frac{C_{1}}{C_{2}^{2}}
\end{gathered}
$$

We will find pressure increment, having differentiated (4)

$$
d p_{i}=-\theta_{i} \cdot K_{B} \cdot N \cdot n \cdot \frac{d r}{\left(r+r_{1}\right)^{3}} \cdot \frac{C_{1}}{C_{2}^{2}}
$$

The internal volume of pressure will change due to the deformation which has resulted because of change of pressure in the pipeline [3]:

$$
V \text { тр }=\pi \cdot r^{2} \cdot 1,
$$

where $\mathrm{d}$ and 1 are the current values of the diameter and length of the pipe section in question, respectively.

The increment in the volume of the pipe is determined by the formula

$$
d V_{\text {тр }}=d\left(\pi \cdot \mathrm{r}^{2} \cdot \mathrm{l}\right)=2 \cdot \pi \cdot \mathrm{r} \cdot \mathrm{l} d r+\pi \cdot \mathrm{r}^{2} \cdot d l,
$$

where $\mathrm{dr}$ and $\mathrm{dl}$ - increment of the radius of a pipe and its length corresponding to pressure increment $\mathrm{dp}$.

We will find a formula for calculation of the reduced factor of volume rigidity of i-braided HPH having substituted the found increments (5) and (6) in a formula (1)

$$
\mathrm{C}_{\text {пр }}=\frac{\theta_{i} \cdot K_{B} \cdot N \cdot n}{\pi \cdot \mathrm{l} \cdot \mathrm{r} \cdot\left(\mathrm{r}+\mathrm{r}_{1}\right)^{3}} \cdot \frac{C_{1}}{C_{2}^{2}}
$$

\section{Conclusions}

The analytical formula of calculation of the reduced factor of volume rigidity of HPH is received. It is required to carry out a series of tests for her confirmation and specification.

\section{References}

1. A.T. Rybak, V.P. Zharov, V.I. Mirnyj, S.A. Zatolokin DTS-2007: Proc. $8^{\text {th }}$ Int. Conf., 1, 66 - 70 (2007)

2. A.T. Rybak, Proceedings of the International Scientific-Technical and Scientific-Methodical Conference: Hydrogasodynamics, hydraulic machines and hydropneumatic systems, 186-189, (2006)

3. A.T. Rybak, I. V. Boguslavskiy, Vestnik of the DSTU, 2, 249-257 (2010)

4. A.T. Rybak, Vestnik of the DSTU, 6, 200-207 (2006)

5. A.T. Rybak, Theory and methodology of calculation and design of drive systems of technological machines and aggregates of agroindustrial complex, thesis abstract of Doctor of Technical Sciences, 39 (2011)

6. A.T. Rybak, I. V. Boguslavskiy, Bulletin of Machine Building., 10,39-46 (2010)

7. A.T. Rybak, Modeling and calculation of hydromechanical systems at the design stage, 167 (DSTU, Rostov n/D, 2006) [in Russian]

8. A.R. Temirkanov, A.T. Rybak, Vestnik of the DSTU, 4, 176-185 (2014)

9. S.A. Zatolokin, A.T. Rybak, Vestnik of the DSTU, 10, 84-90 (2010)

10. S.A. Zatolokin, Perfection of the theory and methods of designing hydromechanical systems with a pumpaccumulator constant-pressure flow source: the author's abstract of the dissertation of the candidate of technical sciences, 18, (2009)

11. A.V. Anisimov, A.I. Butov, V.L. Kondrashev, K.V. Dolgov, K.E. 7th International Scientific and Technical Conference New technologies for controlling the movement of technical objects, 5, 129-134 (2004)

12. G.M. Vodianik, A.V. Anisimov, N.V. Divulin, 6th International Scientific and Technical Conference New technologies for controlling the movement of technical objects, 4, 144-148 (2003)

13. A.T. Rybak, V.P. Zharov, A.V. Korchagin, International Scientific and Technical Conference 
Progressive technological processes in metallurgy and machine building. Ecology and life support. Information technologies in industry and education, 46-49, (2005)

14. A.T. Rybak, V.I. Mirnyj, Izvestiya Institute of Management and Innovation of the Aviation Industry, 3-4, 75-79, (2005)

15. A.T. Rybak, Vestnik of the DSTU,7, 218-223 (2007)

16. A.T. Rybak, A.A. Serdyukov, International Scientific and Practical Conference Innovative technologies in mechanical engineering, 216-218 (2008)

17. A.T. Rybak, O. V. Lyakhnitskaya, E. N. Shein, XInternational Scientific and Methodological Symposium Contemporary problems of multilevel education, 374-381 (2015)

18. A.T. Rybak, O. V. Lyakhnitskaya, Proceedings of the 7th International Seminar System analysis, management and information processing, 62-67, (2016)

19. GOST 6286-73

20. V. A. Lepetov, Chemistry, 350-367 (1976) 